\title{
RUPTURA TEMPORAL Y TEXTUAL EN UN RELATO DE SERGIO PITOL: "ASIMETRÍA"
}

\author{
José L. Nogales-Baena \\ Universidad de Sevilla \\ joseluisnogales@gmail.com
}

RESUMEN: En este artículo se analiza y comenta la relación existente entre las rupturas textuales, temporales y narrativas en "Asimetría", así como su significado. Se considera que la fragmentación, uno de los aspectos esenciales de la teoría y práctica literaria de Sergio Pitol, es llevada al límite en dicho cuento. Esta fragmentación se manifiesta, en un sentido amplio, tanto en la materia narrada como en la organización temporal y en la propia división textual. Con el fin de observar cómo los aspectos formales son indisociables del contenido, se abordan los puntos clave del argumento y se comenta el significado profundo del relato. Asimismo, se hace hincapié en el innegable aprovechamiento técnico de las rupturas temporales y textuales para producir un discurso polifónico y contrapuntístico.

PALABRAS CLAVE: Sergio Pitol; "Asimetría"; fragmentación; ruptura textual, temporal y narrativa; polifonía.

ABSTRACT: This article examines the relation between textual, temporal and narrative ruptures in "Asimetría", as well as its meaning. Fragmentation, one of the essential aspects of the theory and literary practice of Sergio Pitol, is considered to be carried to the highest degree possible in this short story. The fragmentation manifests itself in the narrative and temporal organization, and in the division of the text. In order to highlight the way in which formal aspects are inseparable from the content, this article concentrates on the key points of the argument and analyzes the deep meaning of the story. At the same time the article stresses the undoubted technical skill with which these temporal and textual ruptures are used to produce a polyphonic and counterpoint effect.

KEYWORDS: Sergio Pitol, "Asimetría", fragmentation, ruptures in narrative, polyphony

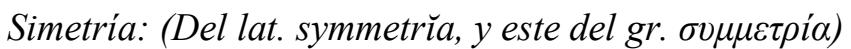

1. f. Correspondencia exacta en forma, tamaño y posición de las partes de un todo.

Los cuatro cuentos de Vals de Mefisto son los más acabados, originales y complejos de Sergio Pitol. ${ }^{1}$ Si bien es cierto que se caracterizan por una serie de temas y procedimientos narrativos comunes, también lo es que cada uno de ellos destaca por su singularidad. Así pues, considero que hay un aspecto esencial de la poética narrativa del escritor que es llevado al límite en "Asimetría":2 la fragmentación, referida esta, en un

1 Vals de Mefisto (1984) es el nombre definitivo del libro publicado anteriormente con el título de Nocturno de Bujara (1981). Los cuentos que lo integran son: "Vals de Mefisto", "El relato veneciano de Billie Upward", "Asimetría" y "Nocturno de Bujara".

${ }^{2}$ De este modo, Alfonso Montelongo no ha dudado en calificar este relato como "emblemático de toda la obra de Pitol" (2007: 98). Firmado en "Moscú, marzo de 1979”, "Asimetría” ganó, ese mismo año, el concurso internacional sobre el cuento de la Universidad Veracruzana. Al año siguiente se publicó en 
sentido amplio, tanto al contenido expuesto de la historia narrada, como a la organización temporal y a la propia división textual.

Dos aspectos complementarios y muy importantes en la teoría y práctica literaria de Sergio Pitol son, por un lado, la idea de que siempre percibimos la realidad de manera parcial y fragmentaria y, por otro, la convicción de que la obra de arte sí puede captar el todo a partir de la representación de sus partes. Aunque estas ideas no han sido formuladas explícitamente, el autor ha recurrido en dos ocasiones a un texto del polaco Jan Kott que alude, a través del tema de la percepción física, al mismo problema: ${ }^{3}$

En la oscuridad el cuerpo estalla en fragmentos, que se convierten en objetos separados. Existen por sí mismos. Sólo el tacto logra que existan para mí. El tacto es limitado. A diferencia de la vista, no abarca la persona completa. El tacto es invariablemente fragmentario: divide las cosas. Un cuerpo conocido a través del tacto no es nunca una entidad; es, si acaso, una suma de fragmentos. (OR 3: 260)

Es en "Nocturno de Bujara", otro de los cuentos de Vals de Mefisto, precisamente, en donde el texto cobra mayor significado. Allí, el narrador comenta: "igual que en el tratado de Jan Kott sobre el erotismo, la fragmentación de la visión podía aplicarse a todo tipo de experiencia sensorial intensa" (OR 3: 263). Así, como ha indicado Laura Cázares, el pasaje "sustenta la propuesta de la fragmentariedad de la percepción y, por lo tanto, la fragmentariedad misma del texto" (2006: 70).

En fin, la voluntad de expresar todas las caras de la realidad narrada por medio de su fragmentación y recomposición selectiva conduce a Pitol, en sus mejores cuentos, a la experimentación constante con el tiempo, el espacio, la voz y la perspectiva narrativa. Esta tendencia culmina con la experimentación a nivel textual en "Asimetría", lo que no volvería a repetirse. En este sentido, esta voluntad expresiva lo acaba ligando a ciertas actitudes de vanguardia, especialmente al cubismo. ${ }^{5}$

Merece recordarse, por tanto, que con Vals de Mefisto el escritor dio por concluida una etapa. Se dedicó a partir de entonces a otros géneros y, con la excepción de "El oscuro hermano gemelo", no volvió a redactar cuentos. De los cinco últimos ha afirmado: "... son indudablemente los mejores, los que mayor felicidad me han proporcionado al escribirlos. A veces pienso que no he intentado hacer otros, porque

Asimetría: Antología personal (1980). Ha aparecido, además de en las colecciones ya mencionadas, en diferentes antologías y recopilaciones posteriores de cuentos, en varias ocasiones con pequeñas modificaciones y correcciones. En este trabajo seguimos la última edición corregida y aprobada por el autor, en Obras reunidas III: Cuentos y relatos (OR 3: 232-49), aunque arrastra y añade algunas erratas.

${ }^{3}$ Los dos lugares son: "Nocturno de Bujara", cuento fechado en 1980; y El mago de Viena (2005).

4 También en "De un diario. 1980", donde Pitol narraba su viaje real a Uzbekistán y la intención de escribir un cuento sobre el lugar, escribió: "Hacer referencia a la traducción que hizo [Juan Manuel] de un texto magnífico de Jan Kott: 'Pequeño tratado de erotismo', que, si mal no recuerdo, trata de la parcialidad de las relaciones en el acto amoroso. Un poco este viaje, y todos los viajes, han sido para mí eso, la aprensión de tres o cuatro imágenes y la imposibilidad de concebir el todo" (Pitol, 1983: 23). El título original del texto polaco es "Mały traktat o erotyce". Se publicó por primera vez en Aloes: Dzienniki i małe szkice (1966). La versión en inglés a la que se alude en "Nocturno de Bujara" es, muy probablemente, la de Boleslaw Taborski en Polish Writing Today (1967). En un artículo anterior he estudiado con mayor detalle el papel que juega el texto de Jan Kott en dicho cuento (véase NogalesBaena, 2014).

${ }^{5}$ En relación a ello escribe Elizabeth Corral: "En 'Asimetría', el trabajo con la forma puede verse aun en la composición tipográfica, en la composición de una segunda columna que nunca volvió a intentar. [...] El lector está obligado a percibir varios planos al mismo tiempo, como sucede en el cubismo, aunque la imagen resultante me hace pensar en las composiciones plásticas construidas con base en diseños geométricos, como las de Mondrián” (2013: 50). 
serían inferiores a estos cinco preferidos, y por eso solo me he interesado en la novela y el ensayo" (OR 3: 29).

Por todo ello, lo que me propongo abordar en este ensayo, sin tratar de agotar la cuestión ni las posibilidades interpretativas del cuento, es la relación existente entre las rupturas textuales, temporales y narrativas en "Asimetría", así como su significado. ${ }^{6}$ No obstante, y dado que ambas están directamente relacionadas con el sentido profundo del cuento, que no es evidente a primera vista, repasaré primero alguno de los puntos claves del argumento.

\section{La esencia del universo es asimétrica}

"Asimetría" comienza in media res con la voz de un narrador en tercera persona que, en estilo indirecto libre, sumerge al lector desde la primera línea en la conciencia y la memoria de Ricardo Rebolledo. Ninguna referencia espacial es ofrecida, el presente de la narración apenas está insinuado, y sólo al final del cuento sabemos que el personaje se encuentra en Veracruz, en casa de su hermana, con quien vive. La distancia entre narrador y personaje oscila a lo largo del cuento, aunque en varias ocasiones sus voces llegan a confundirse. Por ejemplo, en el primer párrafo. He aquí el comienzo:

Apenas logra recordar el inicio de la conversación. De cierto solo sabe que en un momento se levantó, saltó, bailó de alegría para asombro de su hermana, de sus sobrinos y del amigo de su sobrina, a la vez que comentaba que siempre había sabido lo que aquel muchacho sostenía, sí, eso, que el mundo era asimétrico, que la esencia de la materia, de la energía, ¿o qué diablos, de la vida? era asimétrica. (OR 3: 232)

El relato se abre, pues, con la exposición de la "tesis teórica" que sustenta toda la narración: la posibilidad de que el mundo sea asimétrico. ${ }^{7}$ Este descubrimiento, que en principio choca por lo incomprensible, funciona, al igual que en otras narraciones de Pitol, como desencadenante de la conciencia. La acción se centra en lo recordado por Ricardo, mas él no solo recuerda y reflexiona, sino que transmite parte de sus comentarios a sus circundantes, que se convierten en su auditorio. No obstante, todas las cuestiones relativas al presente narrativo quedan entre brumas: el espacio donde tiene lugar la conversación, como ya se ha dicho, la duración del encuentro, la descripción e identidad detallada de los otros personajes, etc.

A partir de ese descubrimiento Ricardo tratará de explicarse su vida o, al menos, el momento que la determinó: la estancia de casi un año que pasó en su juventud en París, en mil novecientos cincuenta y tantos. Viajó a tal ciudad con una beca para estudiar en el conservatorio, pero con la idea real de tener más noticias de su padre, muerto en la capital francesa días antes de la invasión nazi. Sin embargo, ya sin beca y sin haber conseguido averiguar nada sobre su progenitor, Ricardo terminó hospedado en casa de Celeste y Lorenza, dos hermanas mexicanas que, "una marcial y otra mustiamente, envejecían en París" (OR 3: 237).

\footnotetext{
${ }^{6}$ La organización temporal está aún poco estudiada en los cuentos de Sergio Pitol. En este sentido, los trabajos de Mora (2008) y Prada (1996) son fundamentales. Para otros comentarios de "Asimetría" véanse: Acosta (1997: 85-93); Brescia (1998: 188-90); Corral (2013: 50-53); Montelongo (2007); Prada (1996: 94-100); Valdés (1998: 26-34). Como se verá, a lo largo de este artículo aplicamos al cuento una metodología teórica expuesta originariamente para el estudio de la novela - la de Bajtín, por ejemplo, o la de Toro, que completa el modelo formulado por Genette en Figures III (1972) - . Nos basamos para ello en las conclusiones de Mora (1982: 9-56).

7 “Tesis teórica" es el nombre que, en nuestra opinión muy acertadamente, le da Renato Prada (1996: 9495).
} 
Pero, ¿qué significa exactamente que la esencia de la vida es asimétrica?, ¿por qué ese descubrimiento lo explica todo para él?, y, más aún, ¿qué clase de relaciones inconscientes ligan este descubrimiento con la historia de la dos hermanas? Uno de los atractivos del cuento, no el único, reside precisamente en resolver esta serie de cuestiones a las que el propio Ricardo no encontrará respuesta. Él duda desde el inicio sobre si ha comprendido o no el problema planteado, y al final se reafirma en la idea de de que no ha entendido nada. ${ }^{8}$

El significado de "asimetría" viene dado por la negación de su opuesto: la simetría. ${ }^{9}$ El término positivo corresponde al orden, la igualdad y el equilibrio de la forma; mientras que el negativo, a la falta de correspondencia entre sus partes. Desde el comienzo del relato, la equiparación que hace Ricardo entre simetría y forma absoluta es evidente, también su relación con el arte y, de ahí, con la artificialidad de lo simétrico. Si "toda especie animal busca siempre la simetría," razona indirectamente, la esencia asimétrica del universo niega la posibilidad de alcanzarla, lo que convierte su vida en un martirio y un fracaso, ${ }^{10}$ y explica además los más diversos, horribles, excéntricos y singulares hechos de la humanidad: "Eso lo explicaba todo: la fuga de Tolstoi de Iasnaia Poliana, la vasta estirpe de Jack el destripador, los cuartetos de Beethoven, la existencia de Auschwitz, los gestos perfectos de la Dietrich," etc. (OR 3: 232).

Lo que se comprende al final del relato, si el lector une las muchas referencias y pistas sueltas que han ido apareciendo, es que la estancia de Ricardo con las hermanas en París marca un antes y un después en su vida porque encarna, en su imaginario inconsciente, el momento en que consiguió vivir en perfecto equilibrio. Su residencia en la casa está marcada, de hecho, por la armonía establecida entre las dos. Celeste es vigorosa, enérgica, locuaz y docta en literatura; mientras que Lorenza es enfermiza, descuidada, abúlica y entendida en música. La primera habita en la planta alta del hogar, donde cuida hasta la perfección del más mínimo detalle; y la segunda, en la baja, cuya decoración raya en el mal gusto. Ricardo se mueve entre ambos pisos disfrutando de una y de otra más como un agente pasivo que como un auténtico participante de la trama. En la casa siente satisfecha su orfandad y se deja embaucar por la aparente armonía en que coexisten las hermanas. Así, se enuncia en varias ocasiones el valor simbólico que tiene para él ese periodo: "le hicieron sospechar durante casi un año que el paraíso terrenal era posible" (OR 3: 237), "puede decir que conoció el paraíso" (OR 3: 243), "sí, para él fue la representación más perfecta del paraíso" (OR 3: 244).

Sin embargo, a medida que avanza la trama se pone de manifiesto el artificioso pilar sobre el que se sostiene aquella relación. Una serie de maliciosas y oscuras historias del pasado hace presentir la falsedad de ambas con respecto a su tesitura real, llena de odios y rencores ocultos. También, que Ricardo era consciente del engaño, pero prefería ignorarlo. Cuando la situación se hace insostenible para las hermanas, deciden,

\footnotetext{
${ }^{8}$ En el primer párrafo, más adelante de lo ya citado, se dice: "Pero ya en ese momento le comenzó a pesar, por una parte, la certidumbre de que no había logrado comprender de qué hablaba, y, por otra, la sospecha de que toda especie animal busca siempre la simetría" (OR 3: 232). Y en el último: "Y en ese desinterés absoluto por todo lo que turbe su rutina no acaba de entender cómo la conversación con un amigo de su sobrina sobre la hipotética simetría o asimetría de la naturaleza, que ahora está seguro de no haber entendido en absoluto, haya podido dejarlo tan intranquilo" (OR 3: 249).

9 El término "simetría", de origen griego, está formada por el prefijo ov́v- ("con-"), la raíz $\mu c \dot{\tau} \rho \rho v$ ("medida") y el sufijo -i $\alpha$, que significa cualidad. "Asimetría" añade un prefijo de negación al anterior, $\dot{\alpha}$ ("sin").

${ }^{10}$ De hecho, Ricardo, como Lorenza, Celeste y tantos otros, es uno más de los personajes fracasados de Sergio Pitol (véase Brescia, 1998).
} 
como si él fuese el culpable del desequilibrio, despedirlo de la casa. El paralelo con la expulsión del Paraíso está sugerido en el cuento.

En un ensayo posterior, Sergio Pitol ha retomado las mismas ideas en referencia a sí mismo. ${ }^{11}$ La reflexión se abre, igualmente, con la exposición de la tesis teórica: "La física cuántica - aseguran sus intérpretes - ha logrado probar sin demasiado esfuerzo que el mundo, desde su creación hasta hoy, se ha movido a través de un complejo sistemas de asimetrías" (OR 5: 166). De ahí pasa el autor a referirse a la indescifrable y misteriosa vida del universo: “... es el resultado de un juego de difícil comprensión para los legos pero definitivamente cierto y rigurosamente comprobado de formas asimétricas, de fugas de energía hacia lo desconocido..." Después expresa su incomprensión sobre tales relaciones y, finalmente, equipara la simetría con el Paraíso: "Porque, debo confesarlo, mi generación se formó en el culto de la simetría. [...] Pensar en formas simétricas equivale para mí a pasear por los senderos del edén" (OR 5: 16667). ${ }^{12}$

Ahora bien, si toda la esencia del universo es asimétrica y el fracaso nace de la imposibilidad de alcanzar la simetría, como se sostiene en el cuento, también es cierto que se especula en él sobre la posibilidad o no de que las artes - la literatura y la música en concreto - puedan alcanzar la perfección absoluta negada a la existencia. La importancia de esta reflexión es fundamental, pues subyace en el relato, como veremos más adelante, un metadiscurso literario por medio del cual se propone a sí mismo, y al arte en general, como respuesta positiva a dicho dilema.

\section{Fragmentación narrativa, temporal y textual en "Asimetría"}

La ruptura de la tradicional disposición textual es el elemento que a primera vista más llama la atención del cuento. En efecto, "Asimetría" se presenta en forma de segmentos tipográficos mayores separados entre sí por un espacio, y segmentos menores integrados en los anteriores, a izquierda o derecha, enmarcados en blanco. En total, catorce mayores y diecinueve menores. Pueden verse algunos ejemplos a continuación:
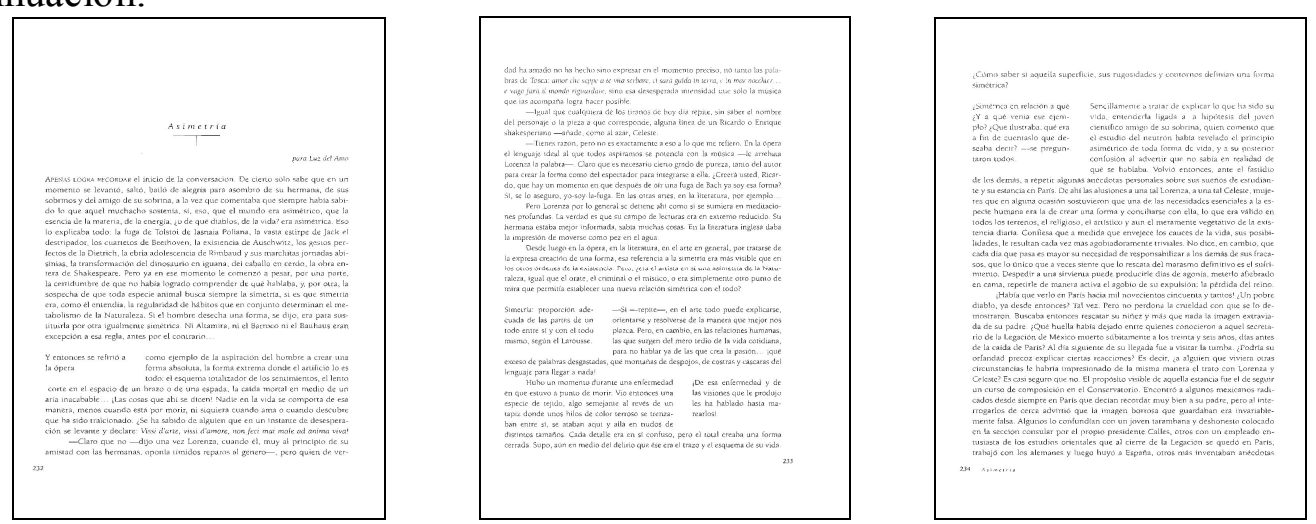

${ }^{11}$ Se trata de un fragmento titulado "De simetrías a asimetrías", en su ensayo Henríquez Ureña visto por sus discipulos, agregado, con modificaciones, a La casa de la tribu en Obras reunidas $V$ (2008), por donde citamos. La versión original se publicó en La Palabra y el Hombre (véase Pitol, 2000), y fue utilizada un año después para los seminarios que impartió en el Tecnológico de Monterrey para la Cátedra Alfonso Reyes (recogida después en Pitol, 2003: 71-93). Por otra parte, el escritor aseguró ya en 1982 que todos los textos de Vals de Mefisto partieron de momentos o anécdotas reales, situaciones o lugares contemplados, y personajes vislumbrados (Pitol, 1982: 14).

12 Compárense estos fragmentos con los ya citados del cuento u otros como el siguiente: "Sencillamente a tratar de explicar lo que ha sido su vida, entenderla ligada a la hipótesis del joven científico amigo de su sobrina, quien comentó que el estudio del neutrón había revelado el principio asimétrico de toda forma de vida, y a su posterior confusión al advertir que no sabía en realidad de qué se hablaba" (OR 3: 234). 
Imagen 1: tres primeras páginas de “Asimetría” en las Obras Reunidas (3: 232-35).

Esta disposición textual, como ya se ha sugerido, no es gratuita, sino que forma parte fundamental de la estructura del cuento y su significado. De hecho, como se ha indicado repetidamente desde comienzos del siglo XX, en la obra de arte toda forma es significante o, en otras palabras, todo significa (Jakobson, Lotman...). Así pues, aunque en ocasiones se haya sugerido que este tipo de juegos tipográficos son más propios de una narrativa infantil, burlesca o humorística (Baquero, 1970: 222-25), en el caso concreto de Pitol se trata de un juego muy serio. De entrada, la ruptura textual introduce la asimetría proclamada por el título, despierta la curiosidad del lector por la novedad ante la que se haya, y llama la atención sobre la composición formal - tema que, más tarde, aparece también en el cuento - . Teresa García Díaz ha explicado que, debido a la disposición textual, el título adquiere de inmediato un carácter remático (2002: 105), y tanto ella como Elizabeth Corral (2013: 50) resaltan la impresión visual que causa el primer encuentro con el texto.

Mientras que la acción principal se desarrolla en los fragmentos mayores, el contenido de los menores es muy variable. Estos apuntan siempre, no obstante, o bien a resaltar o comentar algún aspecto del segmento mayor al que acompañan, o bien a establecer una suerte de diálogo con ellos.

En la mayoría de los casos, lo más común es pensar que estos segmentos expresan, con sus propias voces, el punto de vista de los narratarios de Ricardo, máxime cuando así se indica de forma expresa en el primero de los que aparece en forma de pregunta: “Simétrica en relación a qué? ¿Y a qué venía ese ejemplo? ¿Qué ilustraba, qué era a fin de cuentas lo que deseaba decir? - se preguntaron todos" (OR 3: 234). La historia que cuenta Ricardo, se nos informa, ha sido narrada muchas otras veces, y es cuestionada, ironizada e incluso ridiculizada a través de los comentarios de los fragmentos laterales: "¡De esa enfermedad y de las visiones que le produjo les ha hablado hasta marearlos!” (OR 3: 233), “Todo muy chejoviano?," “Tal, Lorenza!” (OR 3: 238), “¿Lorenza, escarnecida!,” “El Paraíso al fin!” (OR 3: 243).

En otras ocasiones, los segmentos menores cumplen una función meramente informativa. La voz del narrador parece desdoblarse en estos casos, aunque no siempre queda claro quién los formula, como aquel que incluye la definición de "simetría": "Simetría: proporción adecuada de las partes de un todo entre sí y con el todo mismo, según el Larousse" (OR 3: 233). En la mayoría de fragmentos de este tipo se anuncia o resume lo que se está narrando o se va a narrar en el segmento mayor al que acompañan. Por ejemplo: "A partir de ese momento decidió abandonar a su padre" (OR 3: 235) o "Repitió lo que Celeste le dijo sobre los sueños del licenciado González Guito y de las esperanzas depositadas en el futuro de sus hijas" (OR 3: 240).

En fin, es evidente que la ambigüedad en torno a quién pertenecen estas voces y sus puntos de vista es voluntaria. Los fragmentos laterales contribuyen a la ambigüedad del discurso y ofrecen distintas perspectivas desde las que percibir la narración principal. La serie integrada por los apartes constituye, como ha indicado Alfonso Montelongo, "más de un discurso o, en todo caso, su sujeto es múltiple" (2007: 105). Ya sea por el desdoblamiento del narrador o por la opinión expuesta por los narratarios de Ricardo, todos ellos contribuyen al efecto contrapuntístico del cuento, a la ruptura del discurso monológico y al consecuente fortalecimiento de la polifonía. ${ }^{13}$

Lo paradójico es, como señala Montelongo, que también en el interior del discurso principal "hay expresiones que podrían ser apartes, pero no lo son" (2007:

${ }^{13}$ Sobre los conceptos de polifonía y discurso monológico véase el famoso libro de Bajtín Problemas de la poética de Dostoievski (1963). 
106). Esto sucede porque también en los fragmentos mayores la técnica utilizada es la del contrapunto: perspectiva y voz no son monocordes. Como se avanzó antes, la voz del narrador y la de Ricardo confluyen en varios momentos. Además, la narración no responde siempre al punto de vista de este personaje, sino que con frecuencia se desplaza hacia otros. A ello debe agregarse que en la mayor parte del relato no sabemos con exactitud qué es lo que Ricardo solo piensa y qué lo que expone en voz alta. Por último, voces ajenas a las del narrador y Ricardo intervienen también en el relato, tanto en estilo indirecto libre como directo, y sin mediación explícita de ninguno de los dos.

Todo lo anterior está directamente relacionado con la organización temporal porque el desdoblamiento y cruce de voces narrativas de las distintas diégesis suponen de por sí una anacronía. Son dos los niveles diegéticos. El primero, aquel en el que comienza el cuento, el presente de Ricardo y su auditorio, ambos indefinidos pero constantemente presentes. El segundo, un pasado lejano que tiene su núcleo quince años atrás, cuando Ricardo residió en París, y que se ramifica a su vez en varios hilos narrativos. La historia de Ricardo puede dividirse en tres partes: la búsqueda de su padre, la estancia en casa de las hermanas, y la expulsión y regreso a México. La de Celeste y Lorenza puede dividirse en dos: los años de juventud marcados por el fracaso - Lorenza en el mundo de la ópera y Celeste en su relación matrimonial - y la etapa de senectud en que viven en aparente armonía. El eje narrativo en torno al cual gira todo el cuento es el tiempo en que Ricardo vive con las dos hermanas.

El avance de la acción está ligado al desarrollo de los acontecimientos en la conciencia de Ricardo, pero también a la exposición de su discurso a quienes lo están escuchando. El tiempo psicológico del primero es contrapuesto así al tiempo de la experiencia de sus oyentes. ${ }^{14}$ La segmentación tipográfica muestra perfectamente esta relación, pues en algunos puntos los segmentos en forma de pregunta de los que hablamos antes motivan o dirigen la narración. Los fragmentos menores se alejan, además, del punto de vista de Ricardo y se relacionan con el presente. En cambio, los mayores imitan los vaivenes de la memoria y actualizan el pasado. El laconismo de unos frente a la extensión de otros encara el dilatado fluir de la conciencia con la fugacidad de cada instante. Por otra parte, y en tanto que ponen en paralelo los dos niveles diegéticos, participan de un efecto de simultaneidad temporal (Toro, 1992: 45). El pasado reconstruido por Ricardo, el segundo nivel diegético, convive con el presente en una relación dialógica.

En cuanto a cómo avanza la acción a impulsos del flujo de conciencia del personaje, Carmen de Mora ha señalado en referencia a otro cuento del autor: "la estrategia principal seguida por Pitol, en ésta y en otras narraciones, para dicha cuestión consiste en entremezclar el presente narrado con el pasado recordado, el tiempo concreto de la acción con el más abstracto de la introspección, la actividad externa con la interioridad de la conciencia" (Mora, 2008; s. p.). Así, el sistema narrativo de avances y retrocesos es evidente, y, en cuanto se refiere a la duración temporal, se conjugan abruptas aceleraciones y momentos de pausa con multitud de elipsis:

$\mathrm{Y}$ es que la estrategia fundamental seguida por Pitol para configurar el tiempo interno se basa en la tensión entre avances y retrocesos, condensaciones y dilataciones temporales, rupturas cronológicas o anacronías (Genette) que crean un efecto de profundidad y amplifican desde el interior los instantes del tiempo narrado. A veces, largos periodos temporales son aludidos en breves frases, en cambio un momento se dilata durante varios párrafos. (Mora, 2008: s.p.)

${ }^{14}$ Sobre el tiempo interior o psicológico y el tiempo físico o de la experiencia véase Garrido, 1993: 15758 . 
Consecuencia inmediata de todo ello es la confusión entre los dos niveles temporales en el decurso de los fragmentos mayores. El empleo masivo de analepsis y prolepsis da lugar a la superposición y el entrelazamiento temporal explícito (Toro, 1992: 39-40). ${ }^{15}$ En varias ocasiones resulta imposible fijar la cronología de las secuencias narradas y ciertos segmentos pueden ser adscritos a uno $u$ otro nivel. Por ejemplo, el comienzo del segundo fragmento, que en realidad se inicia exabrupto con el primer segmento menor, "Y entonces se refirió a la ópera" (OR 3: 233), puede pertenecer a cualquiera de los dos niveles temporales. Es más, ni siquiera queda claro quién se refirió a la ópera. La intervención de Celeste en estilo directo en el siguiente párrafo y la explicación entre guiones del narrador constatan que "la opera como ejemplo de la aspiración del hombre a crear una forma absoluta" no era una tesis de Ricardo, sino de Celeste. La aparición de las palabras de esta última suponen, además, la evidencia de que en algún punto entre el primer y el segundo segmento se ha saltado del primer nivel diegético al segundo.

Tras cada línea en blanco entre fragmentos, por tanto, puede esconderse un salto temporal, una elipsis y un cambio de perspectiva. La división contribuye a la ambigüedad temporal, a la sensación de discontinuidad narrativa y a las posibilidades de adscribir un mismo discurso a dos o más personajes a la vez. Al comenzar el tercer segmento no queda claro de nuevo si el discurso directo presentado pertenece al primer o al segundo nivel narrativo, a una de las dos hermanas o a Ricardo: "-Sí - repite -, en el arte todo puede explicarse, orientarse y resolverse de la manera que mejor nos plazca. Pero, en cambio, en las relaciones humana..." Además, en el interior de este fragmento vuelve a producirse un salto temporal, un cambio de perspectiva y de acción narrativa. Lo que se narra en el párrafo siguiente, hasta el final del cuento no lo sabremos, corresponde temporalmente a la última parte de la historia de Ricardo, cuando ya ha sido expulsado de la casa:

Hubo un momento durante una enfermedad en que estuvo a punto de morir. Vio entonces una especie de tejido, algo semejante al revés de un tapiz donde unos hilos de color terroso se trenzaban entre sí, se ataban aquí y allá en nudos de distintos tamaños. Cada detalle era en sí confuso, pero el total creaba una forma cerrada. Supo, aún en medio del delirio que ése era el trazo y el esquema de su vida. ¿Cómo saber si aquella superficie, sus rugosidades y contornos definían una forma simétrica? (OR 3: 233-34)

El párrafo es importantísimo porque ofrece una imagen completa del discurso metaliterario que subyace en el cuento: la posibilidad del arte de ofrecer el todo, de crear una estructura cerrada a partir de visiones incompletas de la realidad. Es, también, una metáfora del cuento, tanto de su contenido - la vida de Ricardo, a la que se trata de encontrar sentido -; como de la manera en que técnicamente está compuesto: ese entrelazamiento de voces, historias y tiempos que venimos comentando. Al adelantar esta secuencia en la disposición temporal, el narrador ofrece una pista de lo que el lector

\footnotetext{
${ }^{15}$ La definición que da Fernando de Toro a estos fenómenos es la siguiente: "De un masivo empleo de analepsis y prolepsis resulta la superposición y entrelazamiento temporal explícito. El caso de la superposición se da cuando un personaje fuera de realizar una acción dentro del 'NTI' [nivel temporal I], introduce otro nivel ficticio, por ejemplo, a través de recuerdos que, o pertenecen al pasado ('NT II' [nivel temporal II]) o al nivel de la conciencia (como situaciones imaginadas), y en el último caso, carecen las situaciones de un tiempo cronológico. [...] El entrelazamiento temporal explícito lo encontramos cada vez que un narrador mezcla diversos segmentos accionales de diversas secuencias accionales en forma cronológica o acronológica de tal manera que se produce una especie de mosaico accional" (Toro, 1992: 39-40).
} 
encontrará en el resto del relato y lo previene de nuevo para que centre su atención en los aspectos formales que lo hacen posible. ${ }^{16} \mathrm{La}$ imagen reaparece en el último segmento, cierra la historia de Ricardo con las dos hermanas y recuerda al lector cómo puede interpretar lo que ha leído:

Después ya todo fue lo mismo. En las escaleras de una estación de metro sufrió un desmayo y lo llevaron a un hospital. Mientras deliraba vio ese mapa del que ha hablado, su tejido sinuoso y áspero, y comprendió que era el dibujo de su vida, un espacio de signos ilegibles cuya configuración, independientemente de su voluntad, no desdeñaba la incorporación de ningún elemento, por aberrante que pudiera parecer. (OR 3: 248)

\section{Conclusiones}

Resumiendo, "Asimetría" presenta un discurso fragmentario cuyos componentes principales están en posición dialógica y contrapuntística. El pasado y el presente, el tiempo físico y el psicológico, las voces de unos y de otros se reúnen en el texto y conversan entre sí. El cuento ofrece de este modo, y en la medida de lo posible debido a su brevedad, un discurso polifónico en el que se dan encuentro una pluralidad de voces y conciencias independientes (véase Bajtín, 2012).

El aprovechamiento técnico de la segmentación tipográfica es innegable. En él se apoyan los más variados elementos para ganar sentido, desde el título hasta el significado profundo del cuento, pasando por el zigzagueante vaivén narrativo y la organización temporal. El excelente uso de la elipsis, la alusión y la ambigüedad amplifican el misterio de la narración y dejan su significado abierto a múltiples interpretaciones.

La historia de Ricardo es la de un hombre que trata de encontrar sentido a su vida, pero es también una meditación sobre las posibilidades del arte y la esencia del universo. Se presenta al lector un reto en forma de puzle: una tesis teórica, una reflexión metaliteraria y una serie de historias fragmentarias que deben ser armadas $y$ complementadas para cobrar sentido. Como ha escrito el propio Pitol refiriéndose a algunas de sus lecturas favoritas: "el lector tendrá que recomponer incesantemente una trama que nuca deja de modificarse, donde las aparentes certezas que le permite presentir algunos de los protagonistas quedan parcial o totalmente anuladas por el testimonio del siguiente" (OR 4: 253).

\section{OBRAS CITADAS}

ACOSTA, Salvador (1997): Sergio Pitol y el tema del desencanto: La intertextualidad en su narrativa. Thesis (Ph.D). Los Angeles, University of California.

BAJTín, Mijaíl M (2012): Problemas de la poética de Dostoievski [1963]. Trad. Tatiana Bubnova. $3^{\text {a }}$ ed. México, FCE.

BAQUERO GOYANES, Mariano (1970): Estructuras de la novela actual. Barcelona, Editorial Planeta.

\footnotetext{
${ }^{16}$ Como ya indicamos en la introducción, esta teoría y práctica literaria de Pitol no es exclusiva de este cuento. Carmen de Mora, en referencia a "Vals de Mefisto", lo ha expresado así: "... que el escritor real, Sergio Pitol, no haya querido satisfacer la curiosidad de los lectores por el desenlace implica un reclamo y una exigencia. En lugar de ofrecer una historia completa propone 'visiones esquemáticas' (Ingarden) que aquéllos deberán concretar; al mismo tiempo nos obliga a concentrar la atención en los aspectos compositivos de la historia y de la creación, en general, pues — según he venido comentando- Pitol inserta en este cuento un metadiscurso literario sobre su manera de entender el arte y la escritura" (Mora, 2008).
} 
BRESCIA, Pablo A. J (1998): “"Fracasan, luego existo': Sergio Pitol y sus personajes”, Juan García Ponce y la Generación del Medio Siglo. Coord. José Luis Martínez Morales. Xalapa, Universidad Veracruzana, Instituto de Investigaciones Lingüístico-Literarias, 179-94.

CÁZARES HERNÁNDEZ, Laura (2006): El caldero fáustico: La narrativa de Sergio Pitol. México, D.F., Universidad Autónoma Metropolitana.

CORRAL, Elizabeth (2013): La escritura insumisa: Correspondencias en la obra de Sergio Pitol. San Luis Potosí, El Colegio de San Luis.

GARCÍA DÍAZ, Teresa (2002): Del Tajín a Venecia: Un regreso a ninguna parte. Xalapa, Universidad Veracruzana.

GARRIDO DOMÍNGUEZ, Antonio (1993): El texto narrativo. Madrid, Editorial Síntesis.

MONTELONGO, Alfonso (2007): “Asimetría' en la obra de Sergio Pitol”, Victorio Ferri se hizo mago en Viena: Sobre Sergio Pitol. Coord. Teresa García Díaz. Xalapa, Universidad Veracruzana, 93-107.

MORA VALCÁRCEL, Carmen de (1982): Teoría y práctica del cuento en los relatos de Cortázar. Sevilla, Escuela de Estudios Hispano-Americanos de Sevilla.

(2008): "La herida del tiempo: Lectura de 'Vals de Mefisto' de Sergio Pitol", Ciberletras: Revista de crítica literaria y de cultura, 19 (2008), s.p. http://www.lehman.cuny.edu/ciberletras/v19/moranuevo.html. 17-09-2014.

NOGALES-BAENA, José L. "Intertextualidad en la narrativa breve de Sergio Pitol: "Nocturno de Bujara"", Cuadernos del Hipogrifo. Revista de Literatura $\begin{array}{lllll}\text { Hispanoamericana } & y & \text { Comparada } & 2 & \text { (2014): }\end{array}$ http://www.revistaelhipogrifo.com/wp-content/uploads/2014/11/184-1971.pdf. 16-09-2015.

PITOL, Sergio (1982): “Sergio Pitol: donde sucedió la infancia”. Por Silvia Molina y Rusell M. Cluff. Creación y crítica, 1.6 (1982), 13-15.

(1983): “De un diario. 1980”, Diálogos, 110 (1983), 21-23.

(2000): "Pedro Henríquez Ureña", La Palabra y el Hombre, 113 (2000), 21-34.

(2003): De la Realidad a la Literatura. Monterrey, Instituto Tecnológico de Estudios Superiores de Monterrey.

- (2003-2008): Obras reunidas [abrev. OR] 5 vols. México, D. F.: FCE.

PRADA OROPEZA, Renato (1996): La narrativa de Sergio Pitol: Los cuentos. Xalapa, Universidad Veracruzana, Instituto de Investigaciones Lingüístico-Literarias.

TORO, Alfonso de (1992): Los laberintos del tiempo: Temporalidad y narración como estrategia textual y lectoral en la novela contemporánea (G. García Márquez, M. Vargas Llosa, J. Rulfo, A. Robbe-Grillet). Frankfurt am Main, Vervuert.

VALDÉS MANRÍQUEZ, Hugo (1998): El laberinto cuentístico de Sergio Pitol. Monterrey, México, Consejo Nacional para la Cultura y las Artes.

(C) José L. Nogales-Baena

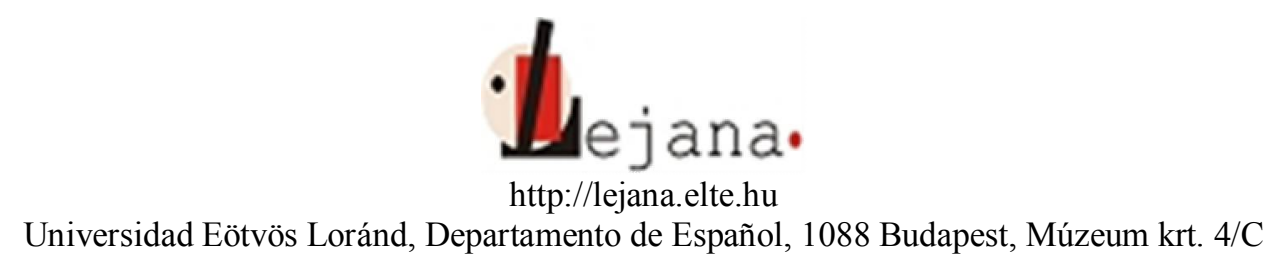

Recibido: 16 febrero de 2015

Aceptado: 13 de octubre de 2015 